\title{
Comparative Efficacy of Atezolizumab plus Bevacizumab and Other Treatment Options for Patients with Unresectable Hepatocellular Carcinoma: A Network Meta-Analysis
}

\author{
Arndt Vogel ${ }^{a} \quad$ Lorenza Rimassa $^{\mathrm{b}}$ Hui-Chan Sun ${ }^{\mathrm{c}}$ Ghassan K. Abou-Alfa ${ }^{\mathrm{d}}$ Anthony El-Khoueiry \\ David J. Pinato $^{f}$ Javier Sanchez Alvarez ${ }^{g} \quad$ Monica Daigl ${ }^{g} \quad$ Panos Orfanos $^{g}$ Michael Leibfried $^{\text {h }}$ \\ Marie-Hélène Blanchet Zumofen ${ }^{g}$ Vincent E. Gaillard ${ }^{g}$ Philippe Merle ${ }^{\mathrm{i}}$ \\ a Hannover Medical School, Hannover, Germany; bedical Oncology and Hematology Unit, Humanitas Cancer Center, \\ Humanitas Clinical and Research Center, IRCCS and Department of Biomedical Sciences, Humanitas University, Milan, \\ Italy; 'Department of Liver Surgery and Transplantation, Liver Cancer Institute, Zhongshan Hospital, Fudan University, \\ Shanghai, China; ${ }^{\mathrm{d} M e m o r i a l}$ Sloan Kettering Cancer Center and Weill Medical College at Cornell University, New \\ York, NY, USA; e USC Norris Comprehensive Cancer Center, Los Angeles, CA, USA; fDepartment of Surgery and Cancer, \\ Imperial College London, London, UK; ${ }^{9}$ F. Hoffmann-La Roche Ltd., Basel, Switzerland; ${ }^{\mathrm{h}}$ Genentech Inc., San Francisco, \\ CA, USA; 'Hepatology Unit, Hôpital de La Croix-Rousse, Lyon, France
}

\section{Keywords}

Hepatocellular carcinoma - Cancer immunotherapy ·

Atezolizumab $\cdot$ Meta-analysis

\begin{abstract}
Background: Most phase 3 clinical trials of systemic therapy for first-line unresectable hepatocellular carcinoma (HCC) have failed, with the exception of SHARP, REFLECT, and IMbrave150. We conducted indirect comparisons of therapies evaluated for first-line HCC treatment. Summary: We conducted a systematic review and meta-analysis of treatments for adults with locally advanced or metastatic unresectable HCC and no prior systemic treatment, including atezolizumab plus bevacizumab, sorafenib, lenvatinib, nivolumab, selective internal radiotherapy (SIRT), transarterial chemoembolization, and placebo or best supportive care. Randomized controlled trials published from January 1, 2007, to March 12, 2020, were retrieved from MEDLINE and Embase. Qualitative assessment of heterogeneity evaluated study designs, pop-
\end{abstract}

ulations, and outcomes. Indirect comparisons used generalized linear models with random effects within a Bayesian framework and informative priors. We calculated relative efficacy estimates with 95\% credible intervals (Crls) and Bayesian posterior probability estimates of atezolizumab-bevacizumab being superior to other treatments. Nine clinical studies with a total of 3,897 participants were identified from 8,783 records and used to build the all-trials evidence network. Indirect comparisons suggested an improved overall survival (OS) with atezolizumab-bevacizumab versus lenvatinib (odds ratio, 0.63 [95\% Crl 0.39-1.04]; with 97\% Bayesian posterior probability of being superior), nivolumab $(0.68$ [95\% Crl 0.41-1.14]; 94\%), sorafenib (0.59 [95\% Crl 0.390.87]; 99\%), SIRT (0.51 [95\% Crl 0.32-0.82]; 100\%), or placebo/best supportive care (0.40 [95\% Crl 0.25-0.64]; 100\%). Key Messages: Within the context of indirect comparisons, analyses of OS favored atezolizumab-bevacizumab versus other treatment options for patients with locally advanced or metastatic unresectable HCC.

(c) 2021 The Author(s)

Published by S. Karger AG, Basel karger@karger.com www.karger.com/lic

Karger $\stackrel{\text { ' }}{5}$

BOPEN ACCESS
(C) 2021 The Author(s)

Published by S. Karger AG, Basel

This is an Open Access article licensed under the Creative Commons Attribution-NonCommercial-4.0 International License (CC BY-NC) (http://www.karger.com/Services/OpenAccessLicense), applicable to the online version of the article only. Usage and distribution for commercial purposes requires written permission.
Correspondence to:

Arndt Vogel, vogel.arndt@mh-hannover.de 


\section{Introduction}

Hepatocellular carcinoma (HCC) accounts for nearly all primary liver cancers and is the fourth most common cause of cancer-related death worldwide [1]. For 10 years, the multikinase inhibitor sorafenib was the only targeted treatment for patients with unresectable HCC $[2,3]$. Several treatment approaches failed to show comparable or improved survival or safety versus sorafenib for first-line treatment. Lenvatinib demonstrated noninferior overall survival (OS) and became a prominent treatment option [4]. In one of the first phase 3 cancer immunotherapy trials for first-line HCC treatment, the single-agent anti-programmed death-1 checkpoint inhibitor nivolumab failed to show an OS benefit versus sorafenib [5]. Single-agent treatment with the vascular endothelial growth factor (VEGF) inhibitor bevacizumab showed clinical and biological activity in a phase 2 trial of unresectable HCC [6].

The IMbrave150 trial investigating the anti-programmed death ligand-1 (PD-L1) inhibitor atezolizumab in combination with bevacizumab demonstrated statistically significant and clinically meaningful OS and progression-free survival (PFS) benefits versus sorafenib for first-line treatment of unresectable or metastatic HCC in patients with no prior systemic treatment [7]. Atezolizumab plus bevacizumab reduced the risk of death by $42 \%$ and the risk of progression or death by $41 \%$ versus sorafenib.

As multiple therapies have become available for HCC, there is a need to understand their comparative benefits and risks. In the absence of head-to-head clinical trials, we performed a network meta-analysis (NMA) to indirectly compare therapies that have been approved for unresectable HCC or have reported first-line treatment data.

\section{Materials and Methods}

\section{Search Strategy and Selection Criteria}

We conducted a systematic literature review of randomized controlled trials studying first-line treatment with any single-agent or combination systemic or locoregional treatment in adults with locally advanced or metastatic unresectable HCC and no prior systemic treatment history. Interventions of interest included combination atezolizumab-bevacizumab, sorafenib, lenvatinib, nivolumab, selective internal radiotherapy (SIRT), transarterial chemoembolization (TACE), and placebo or best supportive care. At the time of this analysis, no other cancer immunotherapy combinations had reported phase 3 results. Nivolumab was included, despite the negative CheckMate 459 trial to help assess single-agent cancer immunotherapy versus the anti-PD-L1/VEGF combination. Locoregional therapies were included as they are recommended first-line options for liver-confined unresectable HCC and remain a treatment in selected regions and patients with advanced HCC.

Cancer Immunotherapy Network Meta-

Analysis for Hepatocellular Carcinoma
We included randomized controlled trials published from the time of sorafenib's US approval for unresectable HCC (2007) to March 12, 2020, in English that met the inclusion criteria described below. Study records were retrieved from MEDLINE (www.nlm. nih.gov/bsd/medline) and Embase (www.embase.com) and were supplemented by a hand search of reference lists from included publications and secondary sources (online suppl. Table 2). Search terms are provided in online suppl. Table 1; for all online suppl. material, see www.karger.com/doi/10.1159/000515302. Records were screened by title and abstract, then full-text was reviewed by 2 independent reviewers with discrepancies adjudicated by a third advisor.

\section{Source Data Analysis}

Summary data were extracted by 1 analyst and $100 \%$ qualitychecked by a second analyst. Two independent reviewers assessed the risk of bias (with discrepancies adjudicated by a third advisor) based on guidance from the Center for Reviews and Dissemination [8].

Eligible studies included at least one outcome of interest: OS, PFS, objective response rate (ORR), duration of response, or adverse events (AEs). ORR was only reported as "confirmed" in 2 publications (CheckMate 459 and IMbrave150) [5, 7]. If a publication did not specify whether objective response by Response Evaluation Criteria in Solid Tumors (RECIST) version 1.1 was measured by independent review facility or investigator assessment, independent assessment was assumed. Safety outcomes included patients reporting any AEs, serious, severe, or treatment-related AEs leading to dose interruption, and AEs leading to treatment discontinuation. For combination regimens, AEs leading to discontinuation were attributed to either treatment component.

Feasibility assessment of the indirect comparisons evaluated the nature and timing of outcome measures across trials. Assessment of heterogeneity in the evidence network included qualitative assessment of study designs, outcome measures, and patient populations. We conducted subgroup analyses to investigate patient characteristics possibly affecting the treatment outcomes when imbalances were observed in the distribution of factors.

Descriptive statistics analyzed patient characteristics and safety outcomes. Indirect comparisons of treatment effects for clinical outcomes were analyzed using a generalized linear model with random effects within a Bayesian framework. For time event endpoints (OS and PFS), the model required the assumption of proportional hazards (constant hazard ratio) to be upheld, verified by graphical inspection of the log-log plots. The traditional random effects approach imprecisely estimates between-study heterogeneity when few studies are available for meta-analysis [9]. We used informative priors for the heterogeneity of treatment effects across trials, given the limited number of studies available to inform each pairwise comparison in our Bayesian random effects approach [10]. Hazard ratios and odds ratios with 95\% credible intervals (CrIs) and Bayesian posterior probability estimates of atezolizumab-bevacizumab being superior to the other treatments were calculated for each indirect comparison [11]. CrIs are also affected by prior belief on the between-trial heterogeneity, instead of relying solely on the trials in the network to calculate the heterogeneity. This can impact the width of the intervals when comparing Bayesian and frequentist frameworks. We analyzed 3 levels of network: i.) all trials, ii.) excluding locoregional therapies, and iii.) including active systemic treatments only. All analyses were conducted using Rversion 3.4.2 and JAGS version 4.3.0, using R2jags (cran.r-project. org). 
Fig. 1. Study selection. Flowchart of study selection. 1L, first line.

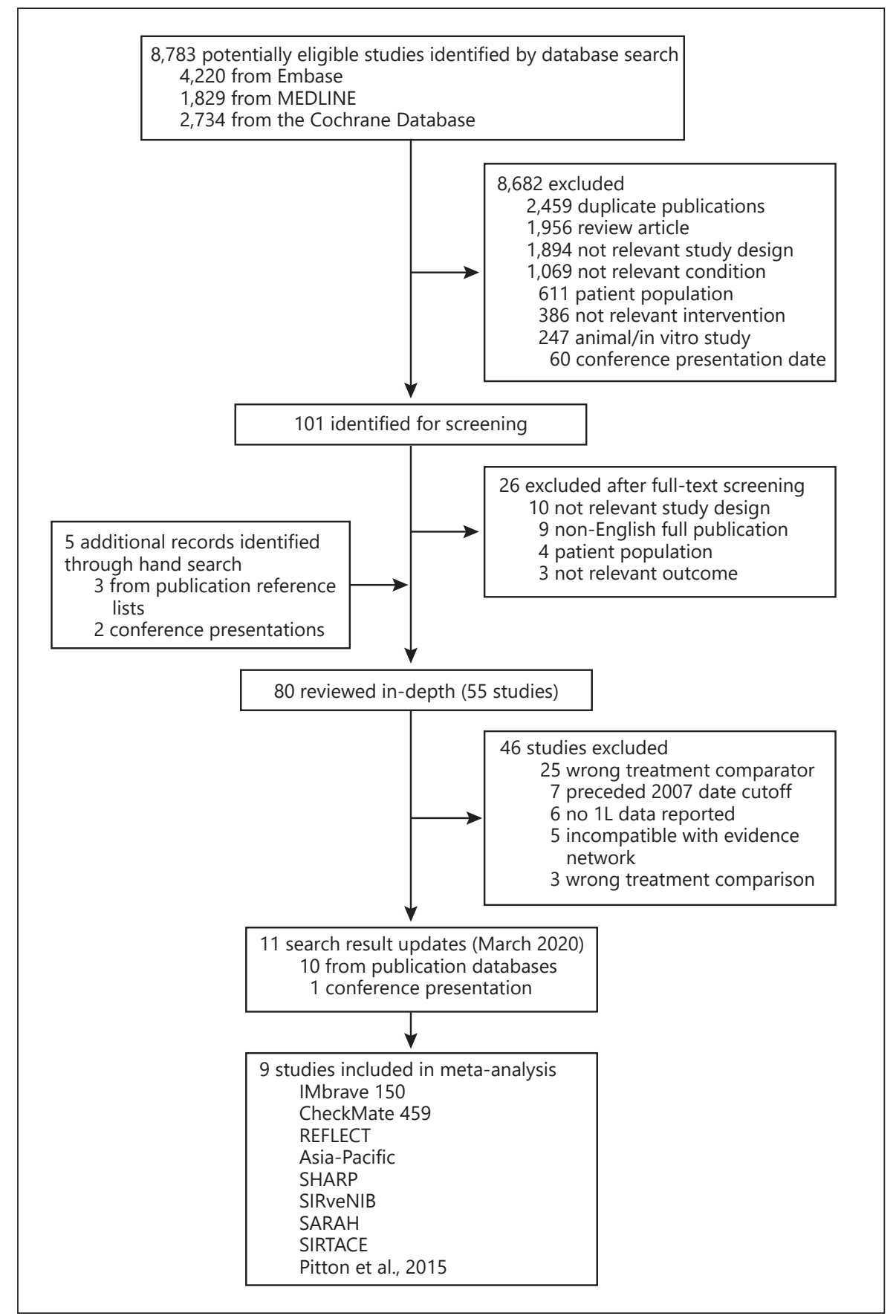

\section{Results}

The database search identified 8,783 records, of which 101 underwent full-text review (Fig. 1). Nine studies (IMbrave150 [7], CheckMate 459 [5], REFLECT [4], AsiaPacific [12], SHARP [2], SIRveNIB [13], SARAH [14], SIRTACE [15], and Pitton et al. [16]), with 3,897 participants constituted the all-trials evidence network (online suppl. Fig. 1). Study and patient characteristics are provided in online suppl. Table 3.

Some differences in study designs, populations, interventions, and outcomes were observed. Median follow-up time was shorter in the IMbrave150 treatment arms (8.9 and 8.1 months) than in REFLECT (27.7 months), CheckMate 459 (15.2 and 13.4 months), SIRTACE (10.7 months), or SARAH (27.9 and 28.1 months; online suppl. Table 3). 
Table 1. Indirect treatment comparisons of clinical efficacy in the all-trials evidence network

\begin{tabular}{llll}
\hline $\begin{array}{l}\text { HR or OR (95\% CrI), probability of } \\
\text { A + B being superior }\end{array}$ & $\mathrm{HR}^{\dagger}$ & $\begin{array}{l}\mathrm{OR}^{\ddagger} \\
\mathrm{ORR}^{*}\end{array}$ \\
\cline { 2 - 3 } & $\mathrm{OS}$ & $\mathrm{PFS}$ & $0.83(0.10-6.94), 39 \%$ \\
$\mathrm{~A}+\mathrm{B}$ versus lenvatinib & $0.63(0.39-1.04), 97 \%$ & $0.91(0.42-1.99), 64 \%$ & $1.17(0.14-9.37), 59 \%$ \\
A + B versus nivolumab & $0.68(0.41-1.14), 94 \%$ & $0.63(0.29-1.41), 92 \%$ & $2.76(0.62-12.65), 94 \%$ \\
A + B versus sorafenib & $0.58(0.39-0.87), 99 \%$ & $0.59(0.34-1.04), 97 \%$ & $0.89(0.10-4.54), 44 \%$ \\
A + B versus SIRT & $0.51(0.32-0.82), 100 \%$ & $0.61(0.31-1.22), 95 \%$ & na \\
A + B versus TACE & na & na & na \\
A + B versus placebo or best supportive care & $0.40(0.25-0.64), 100 \%$ & na & \\
\hline
\end{tabular}

A + B, atezolizumab plus bevacizumab; CrI, credible interval; HR, hazard ratio; na, endpoint not available or reported; OR, odds ratio; OS, overall survival; PFS, progression-free survival; ORR, objective response rate; SIRT, selective internal radiotherapy; TACE, transarterial chemoembolization. * For reporting of objective response, the confirmed responses were used for comparison where available. ${ }^{\dagger}$ Note: $\mathrm{HR}<1.0$ favors $\mathrm{A}+\mathrm{B}$ versus the indirect comparator. ${ }^{\ddagger} \mathrm{OR}>1.0$ favors $\mathrm{A}+\mathrm{B}$ versus the indirect comparator. ${ }^{\S}$ Indirect comparisons with TACE were not feasible because the SIRTACE study (TACE vs. SIRT) did not report ORR according to RECIST 1.1 (mRECIST in Pitton et al. [16]) and did not report either HR for OS or PFS; therefore, the TACE comparison with SIRT could not provide an indirect comparison with the rest of the evidence network through the sorafenib common comparator (via SIRT and sorafenib direct comparison).

Patients in IMbrave150 treatment arms were slightly older (64 and 66 years) than those in REFLECT (62 and 63 years), SIRveNIB (58 and 60 years), or Asia-Pacific (51 and 52 years). Inclusion of patients from the Asia-Pacific region varied across studies. In particular, the REFLECT study included more patients from this region (67\%) than either IMbrave150 (40 and 41\%) or CheckMate 459 (40\%). SIRveNIB and Asia-Pacific included patients only from the Asia-Pacific region, while SARAH, SHARP, and SIRTACE included none. Patients with nonviral etiology were more prevalent in CheckMate 459 (45\%), SHARP (52 and 55\%), and SARAH (61 and 62\%) than in IMbrave150 (30 and $32 \%$ ) or REFLECT (26 and 28\%). A smaller proportion of patients had macrovascular invasion (MVI), extrahepatic spread (EHS), or both in SIRveNIB (30 and 31\%); patients with EHS were excluded from SIRTACE. In CheckMate $459,18-19 \%$ of patients had PD-L1-positive tumors assessed by the PD-L1 IHC 28-8 pharmDx assay (Agilent Dako; Santa Clara, CA, USA). In IMbrave150, 57-64\% of evaluable patients had PD-L1-positive tumors assessed using the PD-L1 IHC SP263 assay (Ventana Medical Systems; Tucson, AZ, USA). Biopsies were not mandatory in IMbrave 150 , and evaluable samples were available in $36.5 \%$ of patients. Region, etiology, MVI, and EHS were heterogeneously distributed and known prognostic factors that may have been effect modifiers.

Some study outcomes were not comparable in the alltrials evidence network (online suppl. Fig. 1). The SIRTACE trial and Pitton 2015 (TACE vs. SIRT) did not report OS or PFS, preventing indirect comparisons of

Cancer Immunotherapy Network Meta-

Analysis for Hepatocellular Carcinoma survival with TACE. Tumor response was assessed by modified RECIST in Pitton 2015, by RECIST 1.0 in SIRTACE, and by RECIST 1.1 in all other trials, preventing indirect comparisons of ORR with TACE; therefore, no estimate of comparative efficacy against TACE could be provided. ORR was measured as confirmed complete or partial response in IMbrave150 and CheckMate 459, and as unconfirmed in other trials reporting ORR, except for in REFLECT (could not be determined).

The risk of bias was generally low across trials. Seven studies had low risk of bias for at least 4 of 7 domains, and risk of bias could not be assessed for CheckMate 459 (inadequate information in the published abstract). Lack of study blinding was the greatest concern for included studies; however, all but 2 trials (Asia-Pacific and SHARP) were open label.

OS is the most objective endpoint when comparing results from different trials and was the primary or coprimary endpoint for most studies. In the all-trials network, indirect treatment comparisons suggested improved OS benefit with atezolizumab-bevacizumab versus lenvatinib (0.63 [95\% CrI 0.39-1.04]; with 97\% Bayesian posterior probability of being superior), nivolumab (0.68 [95\% CrI 0.41-1.14]; 94\%), sorafenib (0.58 [95\% CrI 0.39-0.87]; 99\%), SIRT (0.51 [95\% CrI $0.32-0.82] ; 100 \%)$, or placebo/best supportive care $(0.40$ [95\% CrI 0.25-0.64]; 100\%; Table 1).

Subgroup analyses were conducted to examine population differences in region, etiology, MVI, and EHS that may have affected outcomes. Results by geographic re- 
Table 2. Descriptive assessment of AEs

\begin{tabular}{|c|c|c|c|c|c|c|c|}
\hline \multirow{2}{*}{$\begin{array}{l}\text { Finn et al. [7] } \\
\text { IMbrave150 }\end{array}$} & Atezolizumab plus bevacizumab & 329 & $98.2 \%$ & $38.0 \%$ & $61.1 \%$ & $15.5 \% *$ & $35.0 \%$ \\
\hline & Sorafenib & 156 & $98.7 \%$ & $30.8 \%$ & $60.9 \%$ & $10.3 \%$ & $32.7 \%$ \\
\hline \multirow{2}{*}{$\begin{array}{l}\text { Yau et al. [5] } \\
\text { CheckMate } 459\end{array}$} & Nivolumab & 367 & na & na & na & na & na \\
\hline & Sorafenib & 363 & na & na & na & na & na \\
\hline $\begin{array}{l}\text { Cheng et al. [12] } \\
\text { Asia-Pacific }\end{array}$ & Sorafenib & 149 & $98.0 \%$ & $47.7 \%$ & na & $19.5 \%$ & na \\
\hline \multirow{2}{*}{$\begin{array}{l}\text { Chow et al. [13] } \\
\text { SIRveNIB }\end{array}$} & SIRT & 130 & $60.0 \%$ & $20.8 \%$ & $27.7 \%$ & $2.3 \%$ & $39.9 \%$ \\
\hline & Sorafenib & 162 & $84.6 \%$ & $35.2 \%$ & $50.6 \%$ & $9.9 \%$ & $32.2 \%$ \\
\hline \multirow{2}{*}{$\begin{array}{l}\text { Vilgrain et al. [14] } \\
\text { SARAH }\end{array}$} & SIRT & 226 & na & $77.0 \%$ & na & na & na \\
\hline & Sorafenib & 216 & na & $81.5 \%$ & na & na & na \\
\hline \multirow{2}{*}{$\begin{array}{l}\text { Kolligs et al. [15] } \\
\text { SIRTACE }\end{array}$} & SIRT & 13 & $92.3 \%$ & $53.8 \%$ & na & na & na \\
\hline & TACE & 15 & $66.7 \%$ & $33.3 \%$ & na & na & na \\
\hline Pitton et al. [16] & SIRT & 12 & na & na & na & na & na \\
\hline
\end{tabular}

AE, adverse event; na, endpoint not available/reported; SIRT, selective internal radiotherapy; TACE, transarterial chemoembolization; TEAE, treatment-emergent AE. * AEs leading to withdrawal from any treatment component; 23 patients (7\%) experienced AEs leading to withdrawal from both treatment components.

gion and presence or absence of MVI or EHS were consistent with the overall findings; results were more robust in patients with viral (vs. nonviral) etiology (online suppl. Table 4). The differences by etiology were consistent with those obtained by Casadei Gardini et al. [17] when they compared lenvatinib and sorafenib phase 3 trials.

Findings from 2 sensitivity analyses using restricted evidence networks (i) excluding locoregional therapies (online suppl. Fig. 2) and (ii) focused on active systemic treatments only (online suppl. Fig. 3) showed identical point estimates with increased uncertainty due to the reduced number of data in the evidence network (online suppl. Table 7). Bayesian posterior probabilities of atezolizumab-bevacizumab being superior to lenvatinib, nivolumab, sorafenib, placebo, or best supportive care for OS were all $>90 \%$ in both sensitivity analyses.
Indirect comparisons of PFS suggested improved benefit with atezolizumab-bevacizumab versus nivolumab (0.63 [95\% CrI 0.29-1.41]; 92\%), sorafenib (0.59 [95\% CrI 0.34-1.04]; 97\%), or SIRT (0.61 [95\% CrI 0.31-1.22]; 95\%; Table 1), and moderately improved benefit versus lenvatinib (0.91 [95\% CrI 0.42-1.99]; 64\%). PFS subgroup analyses were not reported for lenvatinib or nivolumab. Relative efficacy results were similar across subgroups for the comparisons with sorafenib and SIRT (online suppl. Table 5). The PFS benefit was more robust with atezolizumabbevacizumab than sorafenib in the HCV and HBV subgroup analyses. Findings from 2 sensitivity analyses using restricted evidence networks showed identical point estimates with increased uncertainty (online suppl. Table 7).

Indirect comparisons in the all-trials evidence network suggested numerically improved ORR with atezoli- 
zumab-bevacizumab versus sorafenib and inconclusive evidence against nivolumab, lenvatinib, or SIRT (Table 1). Relative efficacy results in the subgroups were generally consistent with the primary findings, though limited by the availability of reported subgroup data (online suppl. Table 6). Similar to the PFS findings, 2 sensitivity analyses of ORR using restricted evidence networks showed the same point estimates with greater uncertainty (online suppl. Table 7). Duration of response was not reported in comparators' trials and could not be evaluated.

The comparative assessment of AEs was performed qualitatively. The available safety information from CheckMate 459 was not adequate for comparison (only grade 3-4 treatment-related AEs and AEs leading to treatment discontinuation were reported in the abstract). Occurrence of any AEs was similar with atezolizumabbevacizumab, lenvatinib, and sorafenib (Table 2). In IMbrave150 and REFLECT, patients receiving atezolizumab-bevacizumab or lenvatinib experienced more anygrade serious AEs than patients receiving sorafenib. More AEs leading to treatment changes were also reported with atezolizumab-bevacizumab and lenvatinib versus sorafenib. Fewer AEs were reported with some SIRT or TACE treatment arms than with the systemic therapies. Differences in all-grade serious AEs in studies of SIRT versus sorafenib were not consistent. SIRTACE reported fewer serious AEs in patients randomized to TACE than SIRT. In SIRveNIB, patients randomized to SIRT had fewer AEs, leading to treatment discontinuations than those randomized to sorafenib. Safety results were not reported by subgroup.

\section{Discussion}

This NMA comparing therapies investigated in firstline treatment of patients with locally advanced or metastatic unresectable HCC and no prior systemic treatment history suggested improved OS and PFS with atezolizumab-bevacizumab. Only randomized controlled trials were included as identified in a systematic literature review. NMA was deemed feasible and appropriate to perform indirect comparisons among potential first-line treatment options. Despite the lack of study blinding due to the open-label nature of most studies, 7 of the 9 trials included in the all-trials evidence network used centralized, blinded radiology review. The open-label nature of the trials was not a concern for the survival endpoints but may have influenced other endpoints, such as ORR and

Cancer Immunotherapy Network Meta-

Analysis for Hepatocellular Carcinoma
AEs. Sensitivity analyses assessed the robustness of the results and were generally consistent with the primary findings.

OS provides the most objective assessment of clinical efficacy for indirect comparisons, attributable in part to the uncertainty in ORR assessments across studies due to differences in endpoint definitions (i.e., confirmed vs. unconfirmed). In our analysis, clinical efficacy results remained generally consistent with primary findings when analyzed by subgroups of patients based on the geographic region and presence or absence of MVI, EHS, or both. OS results with atezolizumab-bevacizumab versus sorafenib, lenvatinib, and nivolumab were more robust in patients with viral etiology. Excluding studies investigating locoregional therapies and placebo arms did not affect the main conclusions for systemic treatments; however, greater uncertainty was observed as less information was available to characterize between-trial variability [10]. NMA estimates for indirect comparisons have CrIs broader than the confidence intervals reported in headto-head studies. For the comparison against sorafenib, the estimate from the direct comparison was 0.58 (95\% confidence interval: 0.42-0.79) [7]. This is because NMAs account for the additional source of uncertainty attributable to variation between studies.

ORRs have wide credibility intervals due to the underlying uncertainty in the reporting of ORR using different RECIST methodologies, making inference around the point estimates difficult and limiting their interpretation. Patients deemed to be candidates for locoregional therapies can differ from candidates for systemic therapies; included in indirect comparisons, it is particularly important to ascertain whether their differences may impact treatment performance. Subgroup analyses provided reassurance that regional differences likely did not affect treatment efficacy (online suppl. Table 6). MVI and EHS may have played a role (relative efficacy estimates in the MVI and EHS populations were weaker than the analysis that did not take those differences into consideration). Yet results of relative efficacy in the subgroup with MVI, EHS, or both provided some indication that atezolizumab-bevacizumab may perform better than locoregional therapies in these potential locoregional treatment candidates. Meta-analytic comparisons of safety outcomes can be challenging due to differences in the follow-up time and duration of treatment across trials and treatment arms (e.g., due to deaths and treatment discontinuations). For example, some AEs occurring late (i.e., radiation-induced liver damage) may be difficult to identify and go unreported. Detailed comparisons of toxicity profiles 
were not feasible in this NMA simply comparing reported occurrences of AEs. For this reason, the absolute safety numbers should be interpreted in the context of the differential impact of AEs on patients' lives and the differences in the follow-up time and treatment durations across trials and treatment arms. Issues with toxicity of sorafenib and lenvatinib have been well documented [1820]. In this regard, atezolizumab-bevacizumab provides a different AE profile, reflected in the delayed time to deterioration of quality of life versus sorafenib [7].

This NMA included a robust evidence network of trials. Indirect comparisons suggested more favorable OS and PFS results with the anti-PD-L1 and anti-VEGF combination atezolizumab-bevacizumab than single-agent targeted systemic treatments, locoregional therapies, and placebo or best supportive care. Although more frequent reporting of all-grade serious AEs and AEs leading to treatment discontinuation was observed in trials of atezolizumab-bevacizumab and lenvatinib than sorafenib, which appeared to result in more frequent reporting of AEs and serious AEs than locoregional therapies, the impact of each therapy on patients' lives is not captured in a qualitative comparison of the occurrence of AEs. In contrast to the recent publication by Sonbol and colleagues [21], this NMA focused exclusively on first-line treatment options and included analyses of ORR, safety, and additional patient subgroups. Results for OS and PFS were similar between these 2 NMAs, reinforcing the fact that the atezolizumab-bevacizumab combination appeared to offer the strongest survival benefit.

Our findings should be interpreted with consideration of certain limitations. Full study reports and access to patient-level data would have made the indirect comparisons more robust, particularly for the subgroup analyses that were not reported consistently across trials. Availability of outcome data and consistent outcome definitions would have facilitated additional indirect comparisons (i.e., survival outcomes with TACE). NMAs assume that effect modifiers are similarly distributed in the evidence network; failure to identify such factors and account for imbalances may lead to biased estimation of relative effects. Median follow-up time varied among the studies reporting ORR and AEs: only 8.9 months for patients receiving atezolizumab-bevacizumab in IMbrave150, 27.7 months overall in REFLECT, and 15.2 months for patients receiving nivolumab in CheckMate 459. Due to the current dynamic in the development of new systemic HCC therapies, the use of follow-up treatments differs between studies and may impact OS results - prospective studies are needed to better assess the optimal treatment sequencing. Binary outcomes such as objective response are influenced by follow-up time, where more patients may achieve response when a longer time horizon is used. Similarly, more patients may report AEs over longer treatment exposure and study duration. As locoregional therapies are normally given once, evaluating the impact on discontinuation is difficult. Descriptive assessments of safety were limited by availability and classification across trials, and by differences in treatment exposure and follow-up time. Due to the limited or inconsistent reporting of AEs, safety assessments did not account for the types of AEs nor the impact of AEs on the patient experience. Even descriptive indirect comparisons of AEs can be very sensitive to small differences in reported outcomes; moreover, the frequency of AEs alone is not likely to reflect their impact on patients' well-being. There is considerable variability in the effects of different AEs and their management on patients' clinical status, daily functioning, and quality of life, which was not captured in this descriptive assessment of events due to limited availability. An additional limitation is the evolution of the second-line HCC therapies over time. Both the development of newer tyrosine kinase inhibitors and the emergence of cancer immunotherapies could have impacted the OS in the most recent studies.

\section{Conclusion}

The evidence provided by this NMA supports superior OS and PFS with a manageable occurrence of AEs for atezolizumab-bevacizumab when compared with current TKI, immunotherapy, and locoregional treatment options available for unresectable HCC patients. This study suggests generally consistent efficacy benefit across the subgroups analyzed (geography, etiology, and presence of MVI and/or EHS) when compared to systemic or locoregional treatments. As such, the results of this NMA are in line with recently updated guidelines $[22,23]$ and publications $[7,21]$ that consider atezolizumab-bevacizumab the standard of care for first-line unresectable HCC patients.

\section{Acknowledgements}

Support for third-party writing assistance for this manuscript, furnished by Jeff Frimpter, MPH, of Health Interactions, was provided by F. Hoffmann-La Roche Ltd. 


\section{Statement of Ethics}

The authors have no ethical conflicts to disclose for this systematic literature review and meta-analysis.

\section{Conflict of Interest Statement}

A.V. received honoraria, consulting fees, and speaker bureau fees from AstraZeneca, Bayer, Bristol-Myers Squibb, BTG, Eisai, Lilly, Incyte, Ipsen, Janssen, Merck, MSD, Novartis, Pierre Fabre, Roche, Sanofi, and Servier. L.R. received honoraria and consulting fees from AbbVie, Amgen, ArQule, AstraZeneca, Basilea, Bayer, Celgene, Eisai, Exelixis, Gilead, Hengrui Therapeutics, Incyte, Ipsen, Lilly, Merck, Nerviano Medical Sciences, Roche, Sanofi, and Sirtex Medical; travel expenses from Ipsen; and research funding (to institution) from Agios, ARMO BioSciences, Astellas, AstraZeneca, BeiGene, Eisai, Exelixis, FibroGen, Incyte, Ipsen, Lilly, Merck, and Roche. H.-C.S. received honoraria from AstraZeneca, Bayer, Eisai, Hengrui, Innovent, Johnson \& Johnson, Merck, and TopAlliance, and research funding from Eisai, Hengrui, Innovent, and Roche. G.K.A.-A. received consulting fees from Agios, AstraZeneca, Autem, Bayer, Beigene, Berry Genomics, Celgene, CytomX, Debio, Eisai, Lilly, Flatiron Health, Roche, Genentech, Gilead, Incyte, Ipsen, LAM, Loxo, Merck, MiNA Therapeutics, Polaris, QED, Redhill, Silenseed, Sillajen, Sobi, Therabionics, Twoxar, Vector, and Yiviva, and research funding from ActaBiologica, Agios, AstraZeneca, Bayer, Beigene, Berry Genomics, Bristol-Myers Squibb, Casi, Celgene, Exelixis, Roche, Genentech, Halozyme, Incyte, Mabvax, Puma, QED, Sillajen, and Yiviva; A.E.-K., received honoraria and consulting fees from Agenus, Bayer, Bristol-Myers Squibb, CytomX Therapeutics, Eisai, EMD Serono, Exelixis, Merck, Pieris Pharmaceuticals, Roche, Genentech, Gilead, and QED, and research grants from Merck, Astex Pharmaceuticals, AstraZeneca, and Roche. D.J.P. received lecture fees from ViiV Healthcare and Bayer; travel expenses from Bristol-Myers Squibb and Bayer; consulting fees from MiNA Therapeutics, Eisai, Roche, AstraZeneca; and research funding (to institution) from Merck and Bristol-Myers Squibb. J.S.A., M.D., P.O., M.-H.B.Z., and V.E.G. are employees and shareholders of F. Hoffmann-La Roche, Ltd. M.L. is an employee and shareholder of Genentech, Inc., a member of the Roche Group. P.M. received consulting fees from Bayer, Ipsen, Lilly, Eisai, Roche, AstraZeneca, Bristol-Myers Squibb, and Roche; travel expenses from Bayer, Ipsen, Roche, and Bristol-Myers Squibb; and research funding (to institution) from Ipsen.

\section{Funding Sources}

This study was sponsored by F. Hoffmann-La Roche Ltd. and Genentech Inc., a member of the Roche Group. The study sponsor participated in the design, collection, analysis, and interpretation of data and in the drafting and critical review of the report. The authors had full access to all the data used in the analysis and shared responsibility for the decision to submit for publication. D.J.P. is supported by grant funding from the Wellcome Trust Strategic Fund (PS3416) and acknowledges infrastructural support from the Cancer Research UK Imperial Centre, the Imperial Experimental Cancer Medicine Centre, and the NIHR Imperial Biomedical Research Centre.

\section{Author Contributions}

A.V., L.R., H.-C.S., G.K.A.-A., A.E.-K., D.J.P., J.S.A., M.D., P.O., M.L., M.-H.B.Z., V.E.G., and P.M. contributed to the design and interpretation of findings. J.S.A., M.D., and P.O. conducted the statistical analyses. J.F. wrote the first draft of the report with input from A.V., L.R., H.-C.S., G.K.A.-A., A.E.-K., D.J.P., J.S.A., M.D., P.O., M.L., M.-H.B.Z., V.E.G., and P.M. All authors participated in the writing and editing of the report, and confirm they had full access to all the data in the study and accept responsibility to submit for publication.

\section{References}

1 International Agency for Research on Cancer [Internet]. Global Cancer Observatory. Liver Fact Sheet [cited 2020 Apr 21]. Available from: https: //gco.iarc.fr/today/data/factsheets/cancers/11-Liver-fact-sheet.pdf.

2 Llovet JM, Ricci S, Mazzaferro V, Hilgard P, Gane E, Blanc JF, et al. Sorafenib in advanced hepatocellular carcinoma. N Engl J Med. 2008 Jul;359(4):378-90.

3 Cheng AL, Kang YK, Lin DY, Park JW, Kudo $\mathrm{M}$, Qin S, et al. Sunitinib versus sorafenib in advanced hepatocellular cancer: results of a randomized Phase III trial. J Clin Oncol. 2013 Nov;31(32):4067-75.

4 Kudo M, Finn RS, Qin S, Han KH, Ikeda K, Piscaglia F, et al. Lenvatinib versus sorafenib in first-line treatment of patients with unresectable hepatocellular carcinoma: a randomised phase 3 non-inferiority trial. Lancet. 2018 Mar;391(10126):1163-73.
5 Yau T, Park JW, Finn RS, Cheng A-L, Mathurin $\mathrm{P}$, Edeline J, et al. CheckMate 459: a randomized, multi-center phase III study of nivolumab (NIVO) vs sorafenib (SOR) as first-line (1L) treatment in patients (pts) with advanced hepatocellular carcinoma (aHCC). Annals of Oncology. 2019 Oct;30(Suppl 5):v874-5.

6 Siegel AB, Cohen EI, Ocean A, Lehrer D, Goldenberg A, Knox JJ, et al. Phase II trial evaluating the clinical and biologic effects of bevacizumab in unresectable hepatocellular carcinoma. J Clin Oncol. 2008 Jun;26(18):2992-8.

7 Finn RS, Qin S, Ikeda M, Galle PR, Ducreux M, Kim TY, et al. Atezolizumab plus bevacizumab in unresectable hepatocellular carcinoma. N Engl J Med. 2020 May;382(20): 1894-905.

8 Centre for Reviews and Dissemination [Internet]. Systematic reviews [cited 202016 Jun]. Available from: https://www.york.ac.uk/media/crd/Systematic_Reviews.pdf.
9 Jackson D, Bowden J, Baker R. How does the DerSimonian and Laird procedure for random effects meta-analysis compare with its more efficient but harder to compute counterparts? J Stat Plan Inference. 2010 Apr; 140(4):961-70.

10 Turner RM, Jackson D, Wei Y, Thompson SG, Higgins JP. Predictive distributions for between-study heterogeneity and simple methods for their application in Bayesian meta-analysis. Stat Med. 2015 Mar;34(6):98498.

11 Bland JM, Altman DG. Bayesians and frequentists. BMJ. 1998 Oct;317(7166):1151-60.

12 Cheng AL, Kang YK, Chen Z, Tsao CJ, Qin S, Kim JS, et al. Efficacy and safety of sorafenib in patients in the Asia-Pacific region with advanced hepatocellular carcinoma: a phase III randomised, double-blind, placebo-controlled trial. Lancet Oncol. 2009 Jan;10(1): 25-34.
Cancer Immunotherapy Network MetaAnalysis for Hepatocellular Carcinoma
Liver Cancer 2021:10:240-248 
13 Chow PKH, Gandhi M, Tan SB, Khin MW, Khasbazar A, Ong J, et al. SIRveNIB: selective internal radiation therapy versus sorafenib in Asia-Pacific patients with hepatocellular carcinoma. J Clin Oncol. 2018 Jul;36(19):191321.

14 Vilgrain V, Pereira H, Assenat E, Guiu B, Ilonca AD, Pageaux GP, et al. Efficacy and safety of selective internal radiotherapy with yttrium-90 resin microspheres compared with sorafenib in locally advanced and inoperable hepatocellular carcinoma (SARAH): an open-label randomized controlled phase 3 trial. Lancet Oncol. 2017 Dec;18(12):162436.

15 Kolligs FT, Bilbao JI, Jakobs T, Iñarrairaegui M, Nagel JM, Rodriguez M, et al. Pilot randomized trial of selective internal radiation therapy vs. chemoembolization in unresectable hepatocellular carcinoma. Liver Int. 2015 Jun;35(6):1715-21.
16 Pitton MB, Kloeckner R, Ruckes C, Wirth GM, Eichhorn W, Wörns MA, et al. Randomized comparison of selective internal radiotherapy (SIRT) versus drug-eluting bead transarterial chemoembolization (DEB-TACE) for the treatment of hepatocellular carcinoma. Cardiovasc Intervent Radiol. 2015 Apr; 38(2):352-60

17 Casadei Gardini A, Puzzoni M, Montagnani F, Marisi G, Tamburini E, Cucchetti A, et al. Profile of lenvatinib in the treatment of hepatocellular carcinoma: design, development, potential place in therapy and network metaanalysis of hepatitis B and hepatitis $C$ in all Phase III trials. Onco Targets Ther. 2019 Apr 24;12:2981-8.

18 Li Y, Gao ZH, Qu XJ. The adverse effects of sorafenib in patients with advanced cancers. Basic Clin Pharmacol Toxicol. 2015 Mar; 116(3):216-21.

19 Ikeda M, Kobayashi M, Tahara M, Kaneko S. Optimal management of patients with hepatocellular carcinoma treated with lenvatinib. Expert Opin Drug Saf. 2018 Nov;17(11): 1095-105.
20 Rimassa L, Danesi R, Pressiani T, Merle P. Management of adverse events associated with tyrosine kinase inhibitors: improving outcomes for patients with hepatocellular carcinoma. Cancer Treat Rev. 2019 Jul;77:208.

21 Sonbol MB, Riaz IB, Naqvi SAA, Almquist DR, Mina S, Almasri J, et al. Systemic therapy and sequencing options in advanced hepatocellular carcinoma: a systematic review and network meta-analysis. JAMA Oncol. 2020 Oct;6(12):e204930.

22 National Comprehensive Cancer Network. Hepatobiliary Cancers. Version 5.2020 . Available at: https://www.nccn.org/professionals/physician_gls/pdf/hepatobiliary.pdf. Accessed 2021 Jan 6.

23 Heimbach JK, Kulik LM, Finn RS, Sirlin CB, Abecassis MM, Roberts LR, et al. AASLD guidelines for the treatment of hepatocellular carcinoma. Hepatology. 2018;67(1):358-80. 